\title{
Avaliação do Impacto do Uso de Realidade Aumentada em Atividades Colaborativas em Contexto Industrial
}

\author{
Breno Keller \\ Universidade Federal de Ouro Preto \\ Ouro Preto, Minas Gerais \\ kellerbrenons@gmail.com
}

\author{
Saul Delabrida \\ Universidade Federal de Ouro Preto \\ Ouro Preto, Minas Gerais \\ saul@sdelabrida.com
}

\section{RESUMO}

O advento da Indústria 4.0 criou novas demandas de desenvolvimento, tais como a criação de sistemas interconectados e novas formas de se interagir com esses sistemas. Dessa forma, a Realidade Mista pode ser utilizada como uma ferramenta facilitadora deste processo. Portanto, a combinação da realização de atividades colaborativas em contexto industrial com a utilização de recursos de Realidade Mista como intermediadores da colaboração, se mostra promissora. Logo, o objetivo deste trabalho é avaliar como a utilização de recursos de Realidade Mista impactam na realização de atividades colaborativas na Indústria 4.0. Como resultados parciais, temos o desenvolvimento de uma ferramenta para interação entre pessoas utilizando Realidade Mista e esperamos que os resultados obtidos com a realização de testes de usuário mostrem caminhos e novas estratégias para o uso desses recursos no contexto industrial.

\section{PALAVRAS-CHAVE}

Inspeção Industrial, Estudo de Usuários, Realidade Mista, Colaboração

\section{INTRODUÇÃO}

A Quarta Revolução Industrial ou Indústria 4.0 é um termo utilizado para descrever o processo de automação na indústria, o qual ocorre mediante comunicação entre equipamentos por meio de sistemas ciber-físicos, internet das coisas e aprendizado de máquina. Porém para que esses sistemas possam operar corretamente é necessário o desenvolvimento de novas interfaces que permitam aos operadores interagir com os equipamentos e outros operadores de forma eficiente.

Uma alternativa para essas interações é a colaboração, a qual pode ser definida como a execução de atividades em conjunto. Onde esse conjunto é composto por dois ou mais atores sendo eles: um ser humano ou uma máquina. Dessa forma, é possível caracterizar três cenários: (i) Colaboração Humano-Humano, (ii) Colaboração Humano-Máquina, (iii) Colaboração Máquina-Máquina.

Porém existem contextos onde não é possível existir a automação completa de uma tarefa, isso é, somente máquinas operando de

Permission to reproduce or distribute, in whole or in part, material extracted from this work, verbatim, adapted or remixed, as well as the creation or production from the content of such work, is granted without fee for non-commercial use, provided that the original work is properly credited.

IHC 2019 - WORKSHOP DE TESES E DISSERTAÇÕES, Outubro 21-25, 2019, Vitória, Brasil. In Anais Estendidos do XVIII Simpósio Brasileiro sobre Fatores Humanos em Sistemas Computacionais. Porto Alegre: SBC.

(c) 2019 by the author(s), in accordance with the terms of the Creative Commons Attribution-NonCommercial 4.0 International Public License (CC BY-NC 4.0). forma integrada para realizar uma atividade. Dessa forma a colaboração entre humanos se torna essencial para a boa execução de atividades nesses contextos.

Além disso, na última década diversos dispositivos para Realidade Mista surgiram no mercado e vem se tornando acessíveis para o público geral. Uma vez que a Realidade Mista pode ser definida como a combinação do mundo real com recursos virtuais em diferentes níveis, caracterizando tecnologias como Realidade Aumentada (RA) e Virtualidade Aumentada (VA) [8]. Isto posto, ela demonstra ser uma alternativa para auxiliar na realização de atividades colaborativas, dado que esses equipamento estão sendo utilizados como soluções para os mais diversos problemas em diferentes contextos [5]. Esse processo fez com que avaliações sobre a perspectiva de IHC (Interação Humano Computador) devam ser realizadas, considerando que novos equipamentos tecnológicos são novas interfaces de interação. Alguns exemplos de equipamentos para Realidade Mista são o Google Glass, o Microsoft Hololens, o Gear VR e o HTC Vive.

Desta forma este projeto de mestrado busca avaliar sobre a perspectiva de IHC a seguinte pergunta: "Realidade Aumentada como interface de interação afeta a execução de atividades colaborativas na Indústria 4.0?". Onde esperamos que os resultados obtidos mostrem caminhos e novas estratégias para o uso desses recursos no contexto industrial.

\section{TRABALHOS RELACIONADOS}

Conforme definição apresentada anteriormente sobre colaboração, sabemos que uma atividade colaborativa é composta por dois ou mais atores, que podem ser tanto um ser humano, quanto uma máquina. Porém ao se falar em colaboração envolvendo somente máquinas ou um humano e uma máquina, os principais fatores observados nas atividades avaliadas são o desempenho e a segurança do usuário.

Um exemplo disso pode ser visto no trabalho de Orts-Escolano et al. [9], o qual apresenta um software para a transmissão de projeções 3D realistas em tempo real a serem utilizadas por sistemas de realidade aumentada. Para isso são captadas imagens infravermelhas e coloridas, as quais sofrem algumas modificações por meio de processamentos, buscando remover as imperfeições geradas tanto pelo equipamento, como por objetos móveis como o cabelo de uma pessoa. Além disso, a fim de se oferecer uma melhor imersão do usuário a essa interação, o áudio é captado e reproduzido em três dimensões permitindo que o usuário tenha uma percepção da projeção como uma pessoa realmente presente no espaço.

Nas avaliações para atividades colaborativas entre pessoas, os fatores humanos já são considerados com grande importância, como demonstra o trabalho de Dey et al. [4], onde foi realizado um estudo 
sobre o compartilhamento de informações fisiológicas entre dois colaboradores em um ambiente virtual de forma a oferecer uma melhor empatia entre os usuários. Nesse trabalho os autores avaliam se a apresentação de informações sobre a frequência cardíaca dos usuários impacta em uma melhor avaliação na social presence e quais cenários necessitam de maior colaboração entre os usuários. Os resultados alcançados demonstram que o compartilhamento da frequência cardíaca faz com que se tenha uma maior percepção do usuário com o qual eles estão colaborando em todos os cenários.

Já Kim et al. [7] apresentam uma avaliação sobre o impacto do uso de diferentes recursos visuais em realidade aumentada e virtual para realização de tarefas colaborativas remotas. Para avaliar como esses recursos impactam na colaboração foram utilizadas diferentes tarefas simples: montar um tangram, um origami e um conjunto de LEGO. E para avaliação foram observados os resultados das métricas SUS [1], SMEQ e Co-presence. Os quais demonstram que as soluções que apresentaram a opção de se desenhar contornos (um dos recursos disponibilizados) teve o melhor desempenho ao ponto de vista do usuário.

No trabalho de Cidota et al. [2] foi realizado uma avaliação sobre o impacto de notificações visuais e auditivas para avaliar a noção de presença em colaborações remotas. Como tarefa de estudo foi utilizado um puzzle, pois ele atua como um representação de atividades de alta demanda mental, de forma semelhante as tarefas utilizadas no trabalho de Kim et al. [7]. A solução desenvolvida pelos autores apresenta dois tipos de aviso para ações do usuário remoto, sendo eles: um ícone no campo de visão do HMD utilizado e outra um aviso sonoro. Na avaliação realizada foi observado os resultados para o NASA-TLX [6] e um questionário customizado, os quais demonstram que o uso de ambas notificações oferece uma melhor noção da presença do usuário, como as notificações visuais tendo melhor desempenho.

Piumsomboon et al. [10] avaliam o impacto do compartilhamento de informações de contexto entre usuário de realidade aumentada e realidade virtual para a realização de tarefas simétricas e assimétricas. No trabalho, a tarefa simétrica corresponde à identificação de um objeto virtual no espaço comum aos dois usuários e a tarefa assimétrica à movimentação desse objeto até um objetivo. Um ponto observado é que apesar dos usuários terem o mesmo poder de decisão na realização dessas atividades, usualmente o usuário de realidade virtual assume a liderança. Isso pode ser ocasionado pelo equipamento de realidade virtual oferecer maior liberdade ao usuário. Para se avaliar esse trabalho foram definidos dois tipos de avaliação, uma avaliação objetiva onde são observados fatores como tempo necessário para realizar a tarefa e a quantidade de movimentos necessários. Na avaliação subjetiva são avaliados aspectos de usabilidade por meio do uso do SUS e questões de interação social por meio do método de avaliação social.

Por fim, os trabalhos apresentados demonstram que existem pesquisas envolvendo atividades colaborativas desde a construção de sistemas a avaliações de fatores humanos para os diferentes modelos de interação. Porém, esses trabalhos lidam com problemas simplificados que tem grande capacidade representativa, mas não são aplicações reais. Portanto, este trabalho busca desenvolver uma pesquisa que aborde aspectos que possam ser aplicados na solução de problemas reais da indústria nesta transformação da indústria 4.0 .

\section{OBJETIVOS, HIPÓTESES E RESULTADOS ESPERADOS}

Uma das principais áreas em que a Realidade Aumentada (RA) está sendo aplicada é a indústria [5]. Essa utilização fez com que novas demandas surgissem, englobando desde o desenvolvimento de soluções até como os usuários iriam interagir com essas soluções.

Dentro dessas demandas e problemas que surgiram, esse trabalho busca responder à seguinte pergunta: "Realidade Aumentada como interface de interação afeta a execução de atividades colaborativas na Indústria 4.0?". Onde, esse impacto pode ser definido como os fatores humanos envolvidos na colaboração e o desempenho dos participantes na atividade proposta. E essas atividades podem ser dos mais diversos tipos, como inspeções e manutenções de sistemas.

Para responder esta questão optamos por gerar hipóteses de estudo mais especificas que auxiliam a respondê-la.

- H1: A utilização de recursos de RA resulta em um menor tempo para a realização da tarefa;

- H2: A utilização de recursos de RA resulta em uma maior acurácia na realização da tarefa;

- H3: A utilização de Head Mounted Displays (HMDs) demanda uma menor carga de trabalho do usuário para realizar a tarefa;

- H4: A utilização de HMDs apresenta uma pior usabilidade;

- H5: A utilização de recursos de RA melhora a interação entre os operadores durante a realização da tarefa.

Essas hipóteses representam os principais fatores observados na execução de uma atividade colaborativa: interação, eficiência e empatia. Na Seção 5 apresentamos os detalhes de como estas hipóteses serão verificadas pela execução de testes de experiência do usuário.

Por fim, esperamos que este trabalho nos de resultados suficientes para propor direcionamentos a comunidade científica e técnica no desenvolvimento de aplicações de realidade mista para o contexto da indústria. Como resultados secundários, acreditamos no potencial inovador da solução proposta como alternativa para o uso cotidiano em diversos segmentos.

\section{SOLUÇÃO PROPOSTA}

Para avaliar a pergunta e hipóteses descritas na seção anterior foi definido que a tarefa de estudo será uma inspeção realizada de forma colaborativa. A Figura 1 apresenta uma visão geral de como funcionará a interação entre os dois atores (nesse caso, duas pessoas). Em um modelo no qual um ator representa uma pessoa experiente na realização da tarefa enquanto outro ator representa a pessoa que está realizado a tarefa.

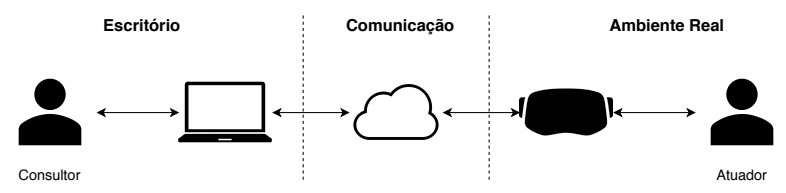

Figura 1: Arquitetura da Solução 
Para realizar essa interação serão considerados recursos de comunicação, tais como: videochamadas e recursos de interação providos por realidade aumentada. Um exemplo da interação por videochamada e RA está disponível no seguinte link: https://youtu.be/ 8LWMj-K7dAc. Esse link apresenta uma demonstração do sistema desenvolvido pelos autores para o Microsoft Hololens, o qual via Realidade Mista insere recursos virtuais para destacar objetos no mundo real. Essa aplicação será utilizada em uma das condições de teste avaliadas que serão descritas posteriormente.

\section{METODOLOGIA}

Para avaliar a solução proposta discutida na seção anterior serão realizados testes de experiência do usuário seguindo a metodologia apresentada na Figura 2. Essa metodologia é baseada em avaliações realizadas na literatura [3].

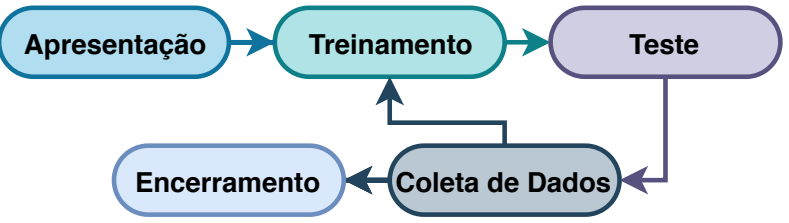

Figura 2: Metodologia para realização de testes de experiência do usuário

A metodologia de avaliação de experiência do usuário que será utilizada é dividida em cinco etapas: (1) Apresentação, (2) Treinamento, (3) Teste, (4) Coleta de Dados e (5) Encerramento. Na etapa de Apresentação, o participante do teste é apresentado ao problema, a tarefa e o procedimento do teste. $\mathrm{Na}$ etapa de Treinamento o participante é apresentado a solução que está sendo validada, para que ele se familiarize com a solução antes da realização do teste. Quando o usuário se sentir confiante a realizar o teste, é realizado o Teste para uma das condições de teste. E após finalizar o teste são coletadas as opiniões dos usuários sobre o que está sendo avaliado para essa condição de teste. Além disso, esse processo se repete enquanto existirem condições a serem avaliadas. Após avaliar todas as condições é encerrado o procedimento de teste.

A atividade a ser executada no Treinamento e Teste será uma tarefa de inspeção com roteiro realizada de forma colaborativa. $\mathrm{Na}$ qual os usuário iram ter como ferramenta de comunicação uma transmissão áudio-visual. Além disso, no momento está sendo avaliado qual será o público participante do teste: operadores da indústria ou público geral. Essa avaliação é necessária, pois é custoso recrutar operadores para participar dos testes e o público participante interfere em qual atividade será realizada durante o Treinamento e o Teste.

De acordo com a metodologia definida, é necessário estabelecer as condições de teste e para isso é preciso que sejam estabelecidas as variáveis independentes (Tabela 1) e variáveis dependentes (Tabela 2) envolvidas na avaliação.

A Tabela 1 apresenta as variáveis independentes definidas para o teste. Essas duas variáveis representam características da arquitetura da solução proposta. Isso é, os recursos de comunicação que serão oferecidos aos usuários durante a atividade e o equipamento para utilizá-los.
Tabela 1: Variáveis Independentes

\begin{tabular}{ll}
\hline \hline Variável Independente & Valores \\
\hline \hline Interação por meio de RA & Sim / Não \\
Tipo de Dispositivo Utilizado & Handheld / Handfree \\
\hline \hline
\end{tabular}

Considerando as variáveis independentes apresentadas, temos quatro possíveis condições de teste, descritas abaixo:

(1) Utilizando dispositivo móvel (handheld) sem interação por RA;

(2) Utilizando dispositivo móvel com interação por RA;

(3) Utilizando dispositivo vestível (handfree) sem interação por RA;

(4) Utilizando dispositivo vestível com interação por RA.

Dentre as condições apresentadas, a condição (1) é considerada o baseline por ser o mais próximo de como essa tipo de tarefa é realizado no ambiente real.

Por fim, as variáveis dependentes (Tabela 2) observadas nessas condições são relacionadas as hipóteses levantadas anteriormente. A tabela apresenta a relação entre as variáveis dependentes, as hipóteses descritas na Seção 3 e os métodos utilizados para que seja possível avaliá-las.

Tabela 2: Variáveis Dependentes, Métricas e Relacionamento com as Hipóteses

\begin{tabular}{lll}
\hline \hline Métrica & Métodos & Hipóteses \\
\hline \hline Tempo para realizar a tarefa & Log Interno & $\mathrm{H} 1$ \\
Acurácia na inspeção & Roteiro de Inspeção & $\mathrm{H} 2$ \\
Carga de trabalho & NASA - TLX & $\mathrm{H} 3$ \\
Usabilidade & SUS & $\mathrm{H} 4$ \\
Colaboração & Autoavaliação & $\mathrm{H} 5$ \\
\hline \hline
\end{tabular}

\section{CONSIDERAÇÕES FINAIS}

O trabalho descrito busca avaliar o impacto de se utilizar recursos de realidade aumentada em atividades de inspeção colaborativas no contexto da Indústria 4.0. Como contribuições, esperamos que os resultados obtidos permitam propor direcionamentos a comunidade científica e técnica no desenvolvimento de aplicações de realidade aumentada para o contexto industrial.

Em relação ao andamento da trabalho, as aplicações necessárias para a realização dos testes estão em processo de finalização e os testes descritos foram enviados para avaliação do CEP da instituição e está no aguardo do parecer da avaliação.

\section{ACKNOWLEDGMENTS}

Os autores gostariam de agradecer a Universidade Federal de Ouro Preto, ao Instituto Tecnológico Vale, ao CNPq e a Capes pelo suporte e financiamento no desenvolvimento deste trabalho.

\section{REFERÊNCIAS}

[1] John Brooke et al. 1996. SUS-A quick and dirty usability scale. Usability evaluation in industry 189, 194 (1996), 4-7. 
[2] Marina Cidota, Stephan Lukosch, Dragos Datcu, and Heide Lukosch. 2016. Comparing the Effect of Audio and Visual Notifications on Workspace Awareness Using Head-Mounted Displays for Remote Collaboration in Augmented Reality. Augmented Human Research (dec 2016), 1-15. https://doi.org/10.1007/s41133016-0003-x

[3] Arindam Dey, Mark Billinghurst, Robert W Lindeman, and J Swan. 2018. A systematic review of 10 Years of Augmented Reality usability studies: 2005 to 2014. Frontiers in Robotics and AI 5 (2018), 37.

[4] Arindam Dey, Hao Chen, Chang Zhuang, Mark Billinghurst, and Robert W. Lindeman. 2018. Effects of Sharing Real-Time Multi-Sensory Heart Rate Feedback in Different Immersive Collaborative Virtual Environments. In IEEE International Symposium on Mixed and Augmented Reality (ISMAR). 165-173. https://doi.org/ 10.1109/ismar.2018.00052

[5] P. Fite-Georgel. 2011. Is there a reality in Industrial Augmented Reality?. In 2011 10th IEEE International Symposium on Mixed and Augmented Reality. 201-210. https://doi.org/10.1109/ISMAR.2011.6092387

[6] Sandra G Hart and Lowell E Staveland. 1988. Development of NASA-TLX (Task Load Index): Results of empirical and theoretical research. In Advances in psychology. Vol. 52. Elsevier, 139-183.
[7] Seungwon Kim, Gun Lee, Weidong Huang, Hayun Kim, Woontack Woo, and Mark Billinghurst. 2019. Evaluating the Combination of Visual Communication Cues for HMD-based Mixed Reality Remote Collaboration. In Proceedings of the 2019 CHI Conference on Human Factors in Computing Systems - CHI '19. ACM Press, 1-13. https://doi.org/10.1145/3290605.3300403

[8] Paul Milgram and Herman Colquhoun. 1999. A Taxonomy of Real and Virtual World Display Integration. In Mixed Reality: Merging real and virtual worlds. 1--26. https://doi.org/10.1007/978-3-642-87512-0_1

[9] Sergio Orts-Escolano, Christoph Rhemann, Sean Fanello, Wayne Chang, Adarsh Kowdle, Yury Degtyarev, David Kim, Philip L. Davidson, Sameh Khamis, Mingsong Dou, Vladimir Tankovich, Charles Loop, Qin Cai, Philip A. Chou, Sarah Mennicken, Julien Valentin, Vivek Pradeep, Shenlong Wang, Sing Bing Kang, Pushmeet Kohli, Yuliya Lutchyn, Cem Keskin, and Shahram Izadi. 2016. Holoportation: Virtual 3D Teleportation in Real Time. In Proceedings of the 29th Annual Symposium on User Interface Software and Technology - UIST '16. 741-754. https://doi.org/10.1145/2984511.2984517

[10] Thammathip Piumsomboon, Arindam Dey, Barrett Ens, Gun Lee, and Mark Billinghurst. 2019. The Effects of Sharing Awareness Cues in Collaborative Mixed Reality. Frontiers in Robotics and AI 6 (2019). https://doi.org/10.3389/ frobt.2019.00005 